\section{NONLINEAR ASPECTS OF \\ SHOCK RESPONSE IN ISOLATED ACCELEROMETERS}

$$
\text { SAND- }-92-0517 \mathrm{C}
$$

DE92 010827

\author{
Thomas L. Paez \\ Experimental Mechanics Department \\ Sandia National Laboratories \\ Albuquerque, New Mexico \\ Norman Hunter \\ Vibration Testing Section \\ Los Alamos National Laboratory \\ Los Alamos, New Mexico
}

\section{Blographies}

Thomas Paez is a senior member of the technical staff in the Experimental Mechanics Department at Sandia Navional Laboratories. He earned his BS and MS degrees in Civil Englneering at the Unversity of New Mexico, and his PhD at Purdue University. He has worked in both education and industry and has nineteen years of experience in random vibration, structural mechanics, and mochanical system testing.

Norman Hunter is a staff member in the Vibration Testing Section at Los Alamos National Laboratory. He earned his BS degree in Electrical Engineering at Mississippi State University and his MS at the Universtty of New Mexico. He has been involved in testing at Los Alamos National Laboratory and Sandia National Laboratories, and has twenty-eight years of experience in mechanical system testing. test analysis and control, and chaotic vibrations.

This investigation was funded by the Department of Energy.

\section{Abstract}

Numerous investigations have studied the potential for chaotic vibrations of nonlinear systems. It has been shown for many simple nonlinear systems, that when they are exclted 88verely enough, or with the appropriate parametric combinations, that they will execute chaotic vibrations. The present investigation considers the potential for the occurrence of chaos in a practical nonlinear system - the isolated accelerometer. A simple, first order model is proposed for the isolated accelerometer, and it is shown that chaos can occur in the isolated accelerometer. A preliminary investigation into the bearing that this chaos potential has on the measurement of shock response is summarized.

\section{Introduction}

Recognition of the possibility of chaotic vibrations of deterministic systems is usually traced to Honrl Poincare [1], French mathematician and philosopher who lived and practiced at the turn of the century. He recognized that the classical equatione of physical systems driven by detorministic axcitaions can vield unpredictable colutions, and thut small changes in the inttial conditions can lasd to tange changes in the final colution of some nonlinear problems. These are somo of the characteristics of the

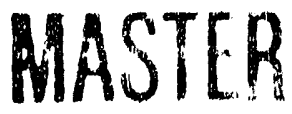


phenomenon that we now call chaos. The fundamental ldeas and some advanced topics in chaotic vibrations are discussed in a number of texts, for example, References 2 and 3.

Chaotic vibrations occur in nonlinear systems with the appropriate parameters, sometimes in response to initial conditions only, and somelimes in response to periodic, deterministic excltations with the appropriate parameters. In typical physical systems, one manifestation of chaos is an aperiodic response to periodic inputs. For example, it will be shown later that some sinusoidal inputs with the appropriate frequencies and amplitudes, excite aperiodic responses in a single-degree-of-freedom (SDF) system with linear viscous damping and stiffness that is guverned by a tangent function.

Another manifestation of chaos in nonlinear systems is known as sensitive dependence on initial conditions. This means that when a response is chaotic, small changes in the initial conditions of the system can lead to large differences in the responses. This characteristic of chaotic response is related to quantities known as the Lyapunov exponents of a dynamic system, and has associated with it a graphic explanation of why chaos occurs. (See Reference 4.) The explanation is outlined here.

Every physical system has equations governing Its motion, and these can be written in a general form as

$$
(z)=g(\{z, t)
$$

where $\{z\}$ is the vector of state variables, and g(...) is a vector function (in general, nonilinear) of system parameters, the state variables and other functions of time (namely, the input). The dot denotes differentlation with respect to the. Equation 1 presints a state space representation, described, ior exsmple, in Reference 7. This representation tracks the response though the space of response measures, we displacement, valocity, etc. When the system parameters are selected and the input is estab. lished Eq. I can be solved to determine the sys- com response. The response that evolves from a specific set of initial conditions establishes a trajectory in the state space of the system.

When the iunction $g(. .$.$) is nonlinear, there is$ assoclated with Eq. 1 a variational equation that - ostablishes a local (in time) linear approximation to the equation of motion. It is denoted

$$
[\dot{z}]=[a]\{z\}
$$

where [a] is the constant coefficient matrix whose th row and jth column element is defined as

$$
a_{i}=\frac{\partial g_{i}}{\partial z_{j}}
$$

where $g_{1}$ is the ith row in the vector function on the right hand side in Eq. 1. The matrix [a] is known as the system Jacobian. Equation 2 indicatses that over a short period of time the response is approximately governed by a linear. first order, ordinary differential equation. The response trajectory over the period of tims governed by Eq. 2 is the single response related to the initial conditions at the beginning of the short time period. The collection of possible responses that might evolve if the initial condithens at the beginning of the short time period were changed by a small amount is governed by the characteristics of the matrix [a]. In parvicular, the evolution of the response relates directly to the eigenvalues of the matrix [a].

To consider this issue grephically, refer to the diagrams in Figures 1a, 1b, and 1c. Consider the potential response trajectories that the system under consideration can execute over the ehort pe.lod of lime when Eq. 2 governs system motion. At the initial time the response is deecribed by a point in the state space (shown by the dot in the circle in each of the figures). The response volves over time to another value (shown by the dot in the ellipse in each of the fic ures). Other initial conditions can be considered, for example, the set of points in an 


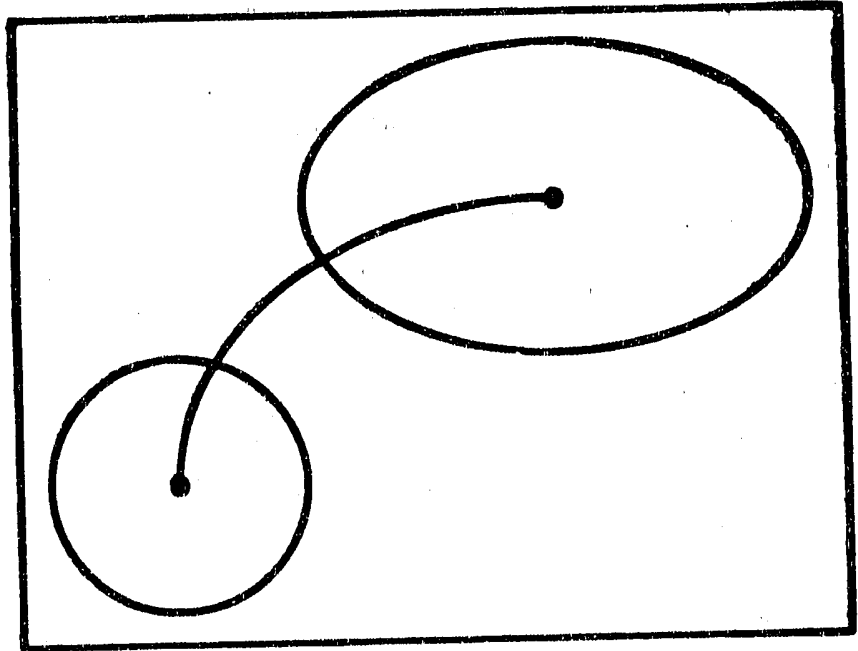

Figure 1a. Unstable state space trajectorios.

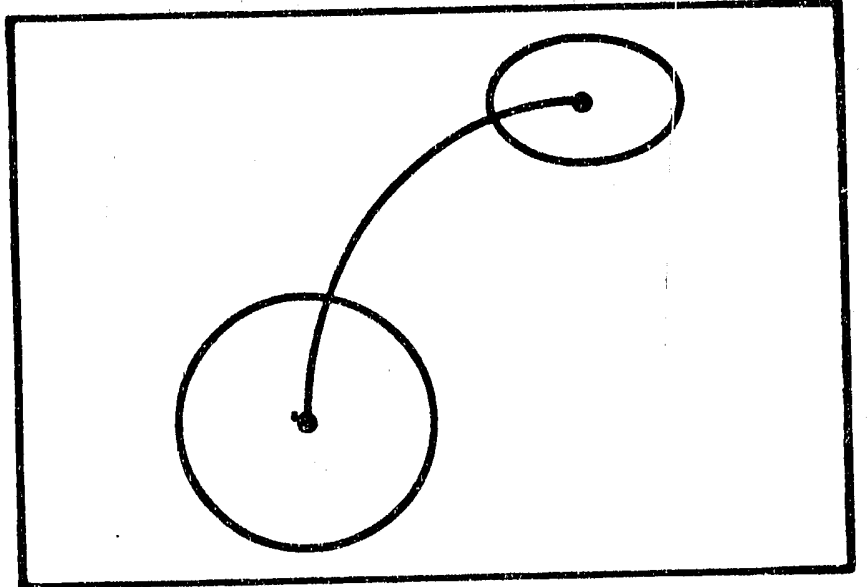

Fioure 1b. Stable state space trafectortes.

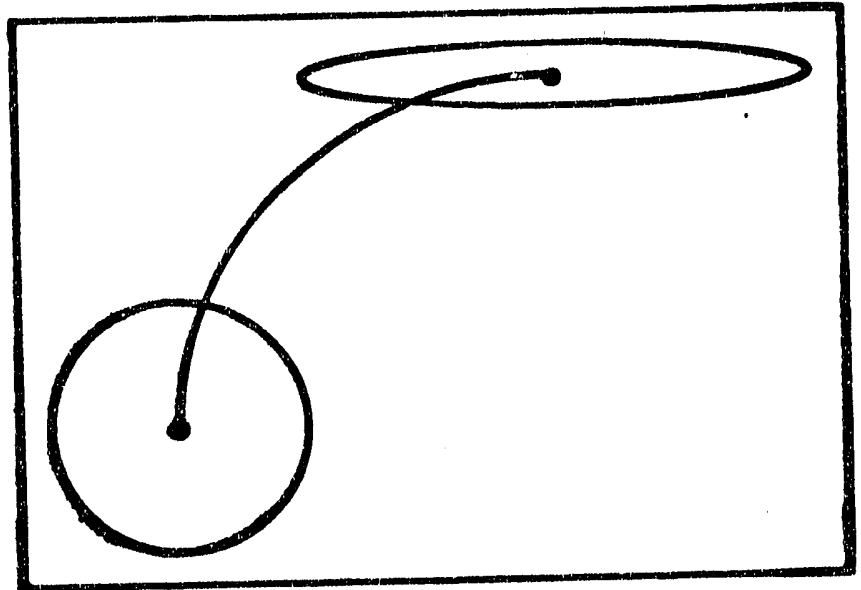

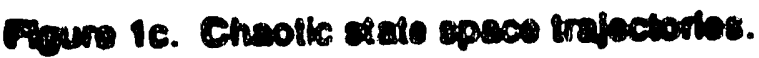

Infinitesimal spherold surrounding the ectual intial condition (shown by clicle in oach figuro). These othor initial conditions would ovolve into other response trajectorles, and would ond up near the actual final point in the iralectory. Epeclifically, they would ovolve into responses in an Infinitesimal ellipsold sur. rounding the actual final point (shown by an olIpse in each of the figures). The trajectories ctaring in an spheriod ovolve into responses raling in an ellipsold because of the linear approximation made in Eq. 2. The relation botween the ellipsold and the spherold establishes the character of eystem response. This lesue nesds to be considered because in real systems finite perturbations to any given trajectory always cocur.

There are three sltultions that can occur. First. It the volume of the ellipsiod is greater than the volume of the spherold then the response is locally (in timo) unstable. In this stiuation, at least one of the eigenvalues of [a] bo postive, and the sum of all the oigenvalues is also postive. If this behavior predominates over a lonf period of time, then the responee of the systern will diverge, I.s., system motion will be unstable. The Lyapunov exponents of the dyniumic aystem quantily the average rate of change of the major axes of the ellipsoid, and thoreby characterize its stability or instability. The sum of the Lyapunov exponents of this system is postive, and roflects the general expansion of trajectories in the state epece.

Second, the volume of the ellipeoid is smaller chan the volume of the apherold and the largest of the major exes of the ellipsold is emaller than the diameter of the spherold, then the response is locally stable. In this stuation, the eigenvalues of the matrix [a] are all negative. If this behavior predominates over a long period of time, then the response of the system will converge, I.e., system motion will be etable. The Lyapunov exponents of this sytem are all negative, and reflect the general contraction of crajoctorive in the state epaos.

Third, the volume of the ellpeoid is emaller 
than the volume of the spheroid and the largest major axis of the ellipsoid is greatep than the diameter of the spheroid, then the response is locally chaotic. In this situation at least some of the eigenvalues of $[a]$ are positive and some are negative. The sum of the eigenvalues is negative. If this behavior predominates over a long period of time, then the response will be chaotic. At least one of the Lyapunov exponents of the system is positive, and one or more are negative. These reflect the long term expansion of trajectories in some directions in state space and the long term contraction of trajectories in other directions in state space.

The potential for chaotic vibrations is a matter of concern in the application of any nonlinear mechanical system. In relation to the isolated accelerometer this potential for chaos means that similar inputs may lead to drastically different accelerometer responses. If chaotic motion occurs during the use of an isolated accelerometer then the acceleration readings do not accurately characterize the measured environment.

This is an important consideration because the use of isolated accelerometers is practically essential in some applications. Specifically, isolation is needed when it is necessary to measure environments that have signal content over a wide spectrum of frequencies which includes the resonant frequency of the accelerometer. When an accolerometor is excited at its resonant frequency it is driven into a nonlinear response range, and it gives an incorrect estimate of the acceleration waveform at all input frequencies.

\section{Equation of Motion for the Isolated Bystom}

In modelling an isolated accelerometer, $i$ is assumed that the isolated accelerometer is constructed by enclosing a standard, commercial accelerometer in an external case. The accelerometer is separated from the external case by an elastic, viscously damped material. The accolerometer case and the external case are assumed to be very stiff relative to the icolating material. It is assumed that the accelerometer is eymmetrically isolated from the extornal case.

The mathematical model to be used to simulate the behavior of the isolated acelerometer is a simple, first order approximation of an actual isolated accelerometer. Specifically, the isoIated accelerometer is modeled as a rigid mass on a nonlinear spring, as shown in Fig. 2 . In the figure, the mass simulates the accelerometer, and the nonlinear spring simulates the material used to isolate the acceleromoter from its external case. The equation governing motion of this system is

$$
\bar{z}=-c \dot{y}-R(y)
$$

where $m$ is the mass of the accelerometer, $c$ is the viscous damping of the isolation material, $R(y)$ is the spring restoring force function, $z$ is the absolute displacement of the mass, $x$ is the absolute displacement of the base, $y=z-x$ is the relative displacement of the mass, and dots denote differentiation with respect to time. Figure 3 shows the restoring force function. It is nearly linear for small displacements, and when the displacement nears plus or minus $y_{m}$ '

the restoring force approaches Infinity (theoretically). The restoring force function is taken to be tangent function in the following. In the model, $y_{m}$ is the thickness of the isolation material.

The equation of motion can be normalized by dividing through by $m$, and expressing $z$ in terms of $y$ and $x$. The iesult is

$$
\ddot{y}+2 \zeta \omega_{n} \dot{y}+\omega_{n}^{2} \frac{2 y_{m}}{\pi} \tan \left(\frac{\pi}{2} \frac{y}{y_{m}}\right)=-\tilde{x}
$$

where $\omega_{n}$ is the natural frequency of the system when it responds to a boy level excitation and $\zeta$ is the viscous damping tactor. 


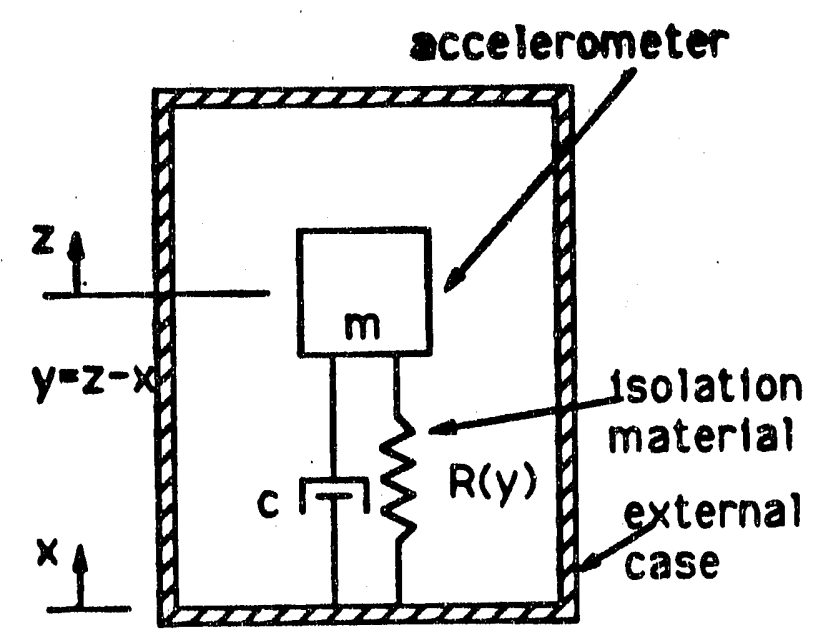

Figure 2. Schematic of isolated accolerometer.

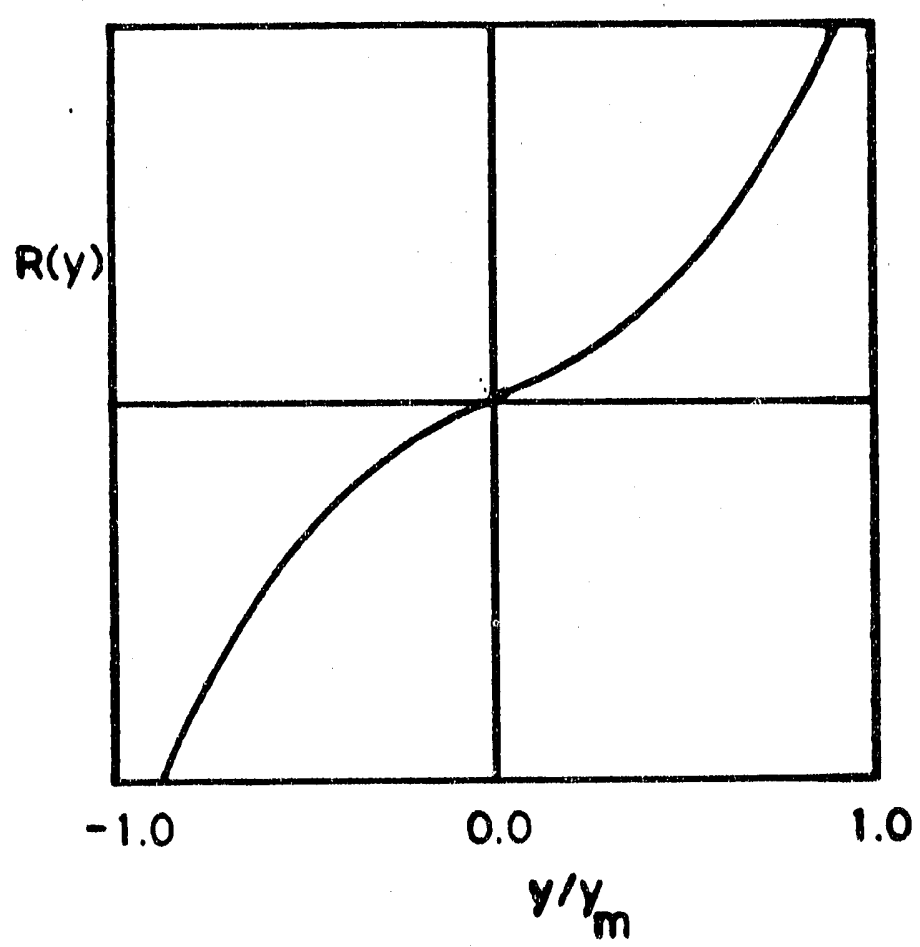

Fipune s. Restoring lonce curve.

In cur meatloation of chaotic vibrations we are interested in considering the response of the oystom to a cinusoldal input. Therolore, we Whe the base acceleration as follows.

$$
-\bar{x}=A \cos \left(\omega_{\rho} x\right)
$$

where $A$ is the amplitude of the input acceleraton and ${ }_{0}$ is its circular froquendy. Substtute Eq. 5 into Eq. 4. The resulting bxpression can be further normalized with respect to time by setting

$$
s=m_{0} t
$$

The reason we have chosen to use $\omega_{p}$ in the temporal normalization instead of $\omega_{n}$ ts that we are Interested in studying the behavior of the cong-term response, and if it is periedic, its period will be an integer multiple of the period of the input. When Eq. 6 is used in Eq. 5 and the resulting expression is divided by $\omega_{1 p}{ }^{2}$ the result is

$$
y^{*}+\frac{2 \zeta}{\alpha} y^{\dot{\alpha}}+\frac{2 y_{\alpha}}{\pi \alpha^{2}} \tan \left(\frac{\pi}{2} \frac{y}{y_{\alpha}}\right)=\frac{A}{\alpha_{0}^{2}} \cos (\tau)
$$

where $\alpha=\omega_{p} / \omega_{n}$ is the ratio of input irequenoy to system small dispiacement natural frequency, and a prime denotes differentlation with respect to $\tau$.

We can normalize the equation with respect to response amplitude by dividing through by the ebsolute value of the maximum potential displacement, $y_{m}$. The resulting oquation is

$$
Y^{\prime \prime}+\frac{2 \zeta}{\alpha} Y^{\prime}+\frac{2}{\operatorname{son}^{2}} \tan \left(\frac{\pi}{2} Y^{\eta}\right)=B \cos (\tau)
$$

chere $Y$ to the ratio of actual displacement to maximum potential displacement and

$$
B=\frac{A}{\omega_{\rho}^{2} y_{m}}
$$

is the normalized input ampillude. Equation 8 t5 the equation wo investlgate in our search for cheos in the motion of an seoleted sccelorometer. 


\section{The Shooting Technique for Chaos Dotestlon}

There are several approaches to establish wheteher or not chaos occurs in system. Among the most direct is the simple observation of the response (or some measure of the response) to specific initial conditions and sinusoidal input over a long period of time. When the response is aperiodic, then the response is in a chaotic regime. However, it is not always clear when chaos occurs when we directly observe the response because in sume situations many cycles of periodic response occur belore the system enters a chaotic regime, and in other situations it takes many cycles for the initial transient to die out belore the periodic response is realized.

To avoid this problem we can directly seek periodic solutions to an equation of motion to establish their existence. If we can show that they do not exist then there is chaos. The shooting technique is a method for seeking periodic solutions to an equation of motion. In the application of this technique we start the system response with arbitrary initial conditions and compute the solution over one period of the sinusoidal input. We compare the final state of the system in state space to the initial conditions and modify the initial conditions until the initial conditions and final state in the state space match as closely as possible. (We use a minimization technique to accomplish this.) If the initial conditions and final state match identically then the olution is called "one periodic." It is called one periodic because the system executes one period of response during one period of the input. (Note that an identical match for the exact oquation is impossible because of computation roundoff and the fact that wo must use an approximate method to compute the solution of the nonlinear differential equaibn. Therefore, fudgement must be used in deciding whether a match oxists.)

If a periodic solution bs not found in the manner described above then we repeat the procedure where we start the system response with arbiirary Initial conditions and compute the solution over two periods of the sinusoldal input. If a periodic soultion is found then it is called two periodic" for obvious reasons. While a perlodic response is not found we repeat the procedure seeking at each step the $n$ periodic response, where $n$ increases to some reasonable limit. If a periodic response is established then this characterizes the system in some sense. If none is found then the response is assumed to have achieved or to be approaching a chaotic state. That is, the Input and system parameters in the vicinity of the ones being tested yield a chaotic solution.

A combination of the shooting technique and the direct observation of system responses was used 10 assess the potential for chaos in the mathematical model for the isolated acelerometer. The existence of chaos was studied for a collection of parameters covering the grid shown in Fig. 4. The damping value chosen for the investigation is ten percent. At each of ten $\alpha$ values and each of ten B values shown on the grid, periodic solutions were sought. Whether or not one was found was noted, and the area on the graph that is shaded denotes the area swhere no periodic solutions were found. It is assumed that system responses are chaotic when the input and system have parameters in this region. Figure 5 shows an example of a poriodic solution, and Fig. 6 shows an example of a cha. otic solution.

\section{Shock Response of the lsolated Accolorometer}

In order to consider in detail the shock response of an bolated accelerometer, we consider the system of Eq 8. Simulation of these equations is accomplished using forth order Aungo-Kutta aborithm with variable stepsize.

We Hustrate the complex nature of the IIme history response of an reolated accelerometer by considering the following dive. 


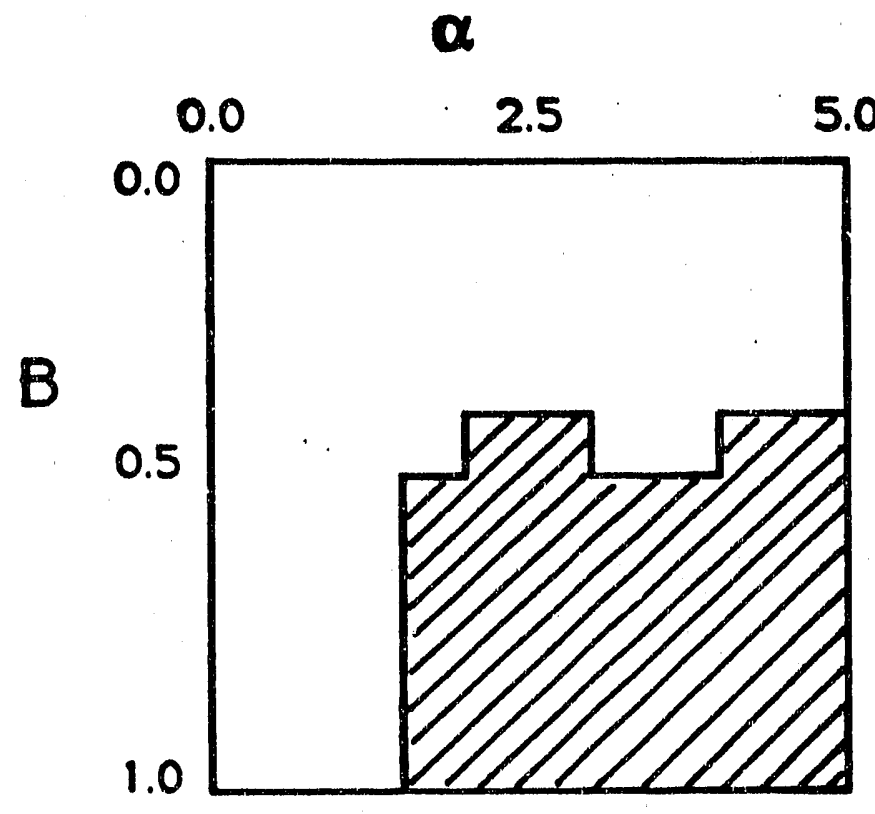

Figure 4. Chaotic region of the normalized, teolated accelerometer.

$$
\begin{array}{rlr}
\bar{x} & =0.2 \sin \left(\frac{1}{4} \frac{2 \pi t}{T}\right)+g \sin \left(4 \frac{2 \pi t}{T}\right), 0 \leq t \leq 2 T \\
& =0 . & \text { elsewhero }
\end{array}
$$

Here $T=2 \pi / \omega_{n}$ is the normalized natural period of the accelerometer, so we are oxching the system with inputs of $1 / 4$ the natural \&requency and four times the natural frequency, simultaneously. We desire to measure only the lower frequency at 1/4 the natural irequency while "isolating", or flitering out, the higher irequency. In the confext of chaotic response to this input the crttical parametor the gain factor 0 which multiplles the higher frequency term. Three cases are considered: $g=0.2$ where the response clearly nonchaotic, $0=3.2$, when the response cloarty chaotlc, and 0.8. where the reaponse is not chaotlc, but the cockicration response dose not nccureteby rollect the mature of the lower fisequency torn Eq. 0.

in the firt cace, we oxctle the bolated

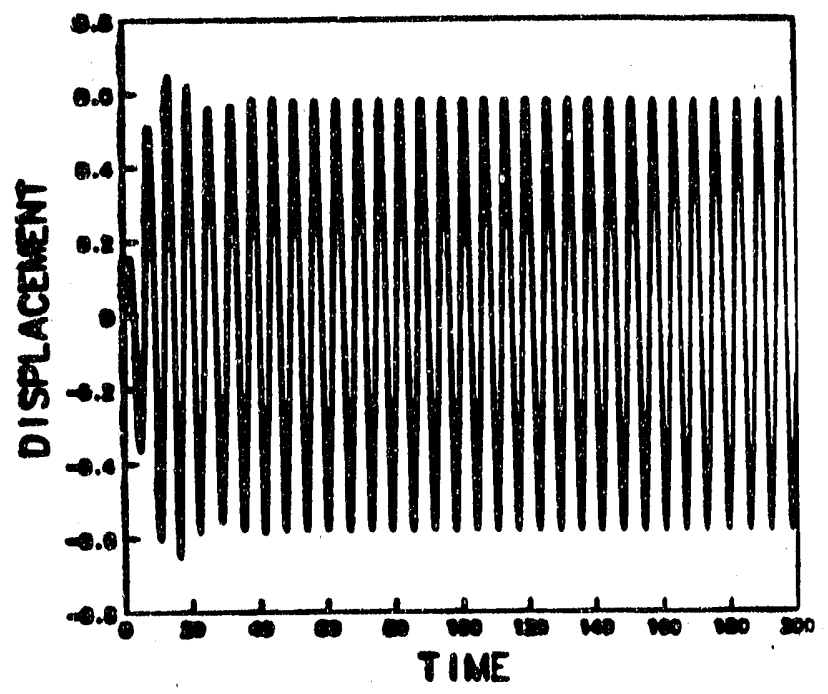

Figure 5. Periodk response for $\alpha=1.0, B=0.2$.

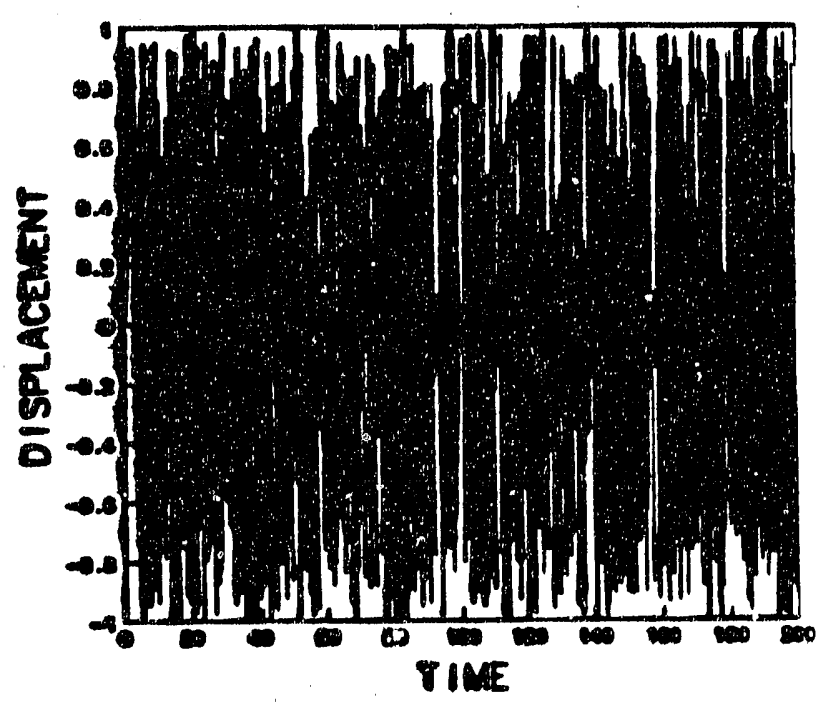

Feure 6. Cheotle respones for $0-3.5,8=0.6$.

eccoleromoter with an umwanted waveform at four tin:es lis natural frequency and aaln, 9. of 0.2. This excitation maintains the accelerometer inside the prodominantly linear responce range and results in the response thown in Fig. 7a. We expect so obtain only the mall sine extending from Too to Ta2.0. This is undeed noest of the respones. wough come contamination cccure scom excitation of the ratural frequency at To2.0 and from the Input 
at four times the natural frequency at Te2.0. In a typleal analysis case, the measured response would be fittered at a frequencoy below the accelerometer's natural frequency. The offects of this filtering (at 0.5 limes the natural (requency) are lliustrated in Fio. 7b. We pudge this approximation of the haff sine input to be casoneble one.

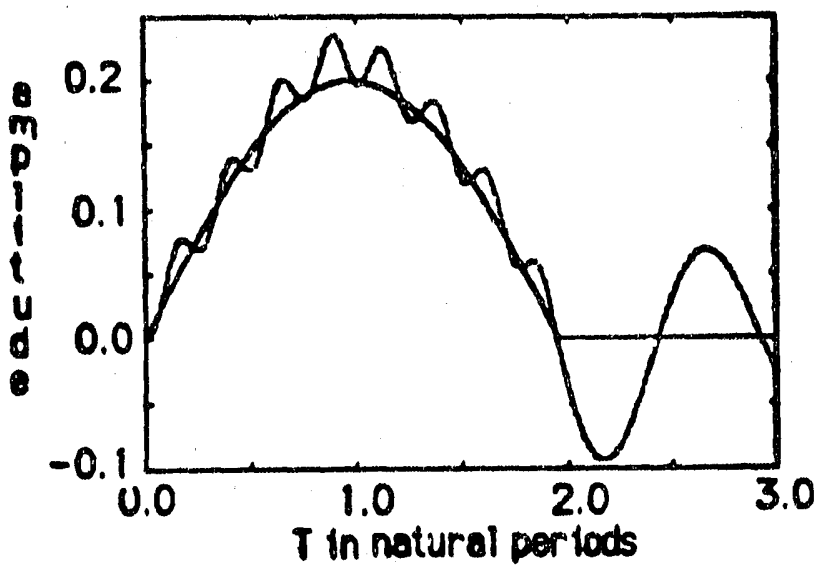

Figure 7a. Input eccoleration and respones a colated accoloromoter for 9-0.2.

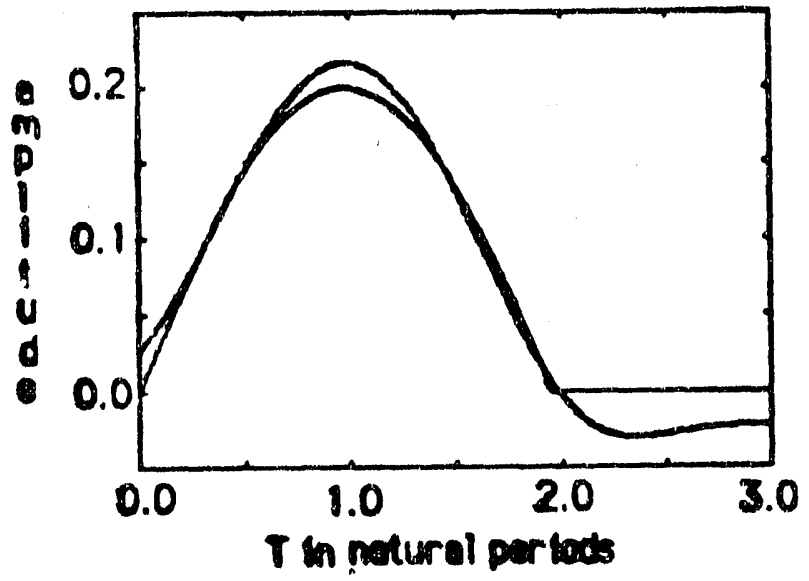

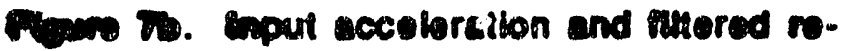

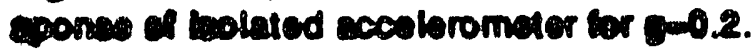

Foures an and ob lllustrafo the recults whon the galn is increased to 3.2 , a fector 16 insos higher than that uced in the previous cese. The unilitered mefonse clearly indicates very nonlinear behavior in the moasured acceleration response. In fect, we have exched the eccolerometer to levels where the displacemente are close to the maximum allowed displacemente (near the barrier, which in a practical case, may be about 0.01 inch from the acceleromoter in the aullibrium posttion) and the stifiness is quite high. Large stifiness values result in the high acceleration peaks evident in Figure 8a. This strongly nonilinear response means that low pass fittering cannot recover the low frequency input waveform. The low pass filtered responsa is compared to the desired response in Figure 8b. Unilke the previous case. where the gain to 0.2 , at these excltation levels, both the shape and magnitude of the acceleration response differ signifiantly from the desired response.

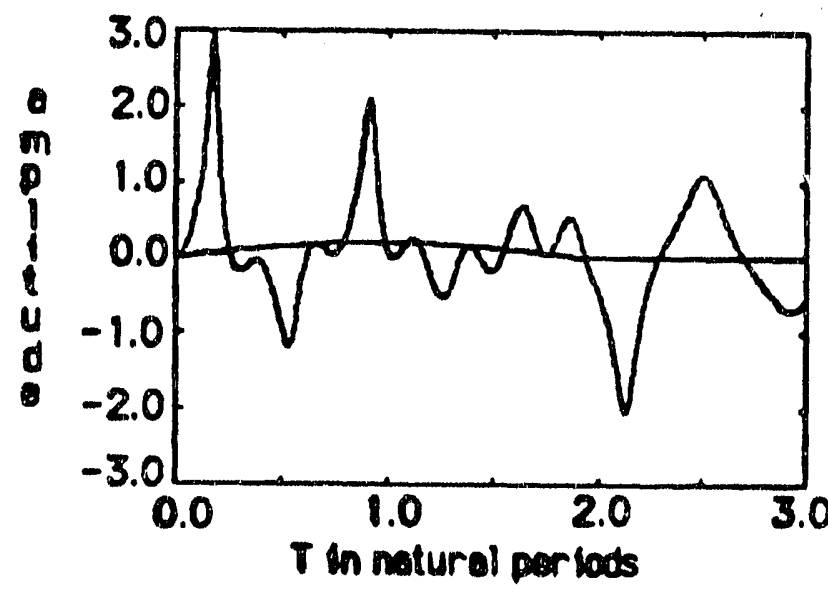

Floure ea. mout accaleration and responese of solatod accoloromoter for gem.2.

mput levels intermediate to those shown above chow progressively oreater degrees of distortion botween the moasured wavelorm and the desired wavelorm. The character of the dynemic behavior of the icolated acceleromeler eystem is invectigated by analyzing the behavior of the input and response time sories. (See roferences $s$ and 6.)

The silect of the belated eccolerometer syctem on en ininitesimal error in the state to shown in Figs. 8a and Ob for the exctation bevals 


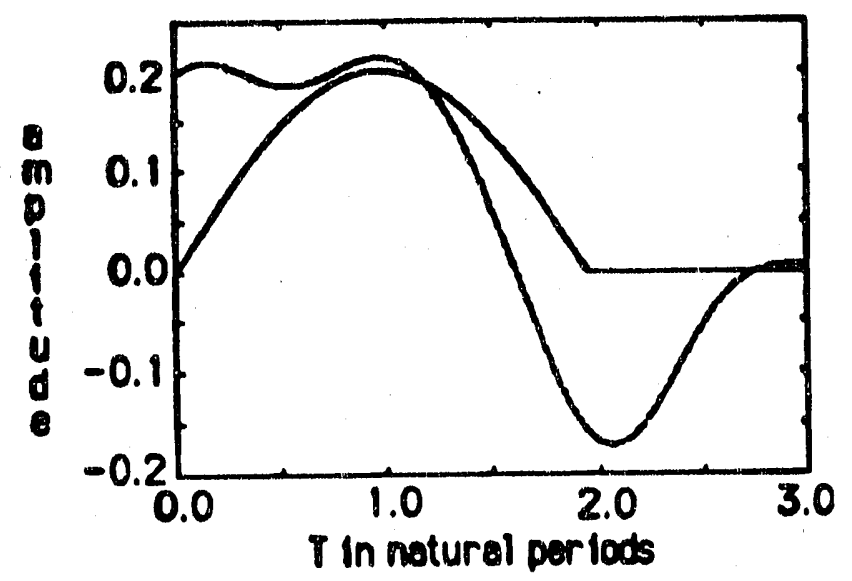

Figure ab. Input acceleration and fittered reeponse of isolated acceleromoter for g-3.2.

corresponding to gains of 0.2 and 3.2. As expected, gain of 0.2 corresponds to a nonchaotic case, where the error decays at a rate approximately consistent with a system damping coofficient of 0.10 . A gain of 3.2 corresponds to a case where the estimated Lyapunor exponent is slightly positive, indicating that the isolated accelorometer is bohaving in a chaotic manner.

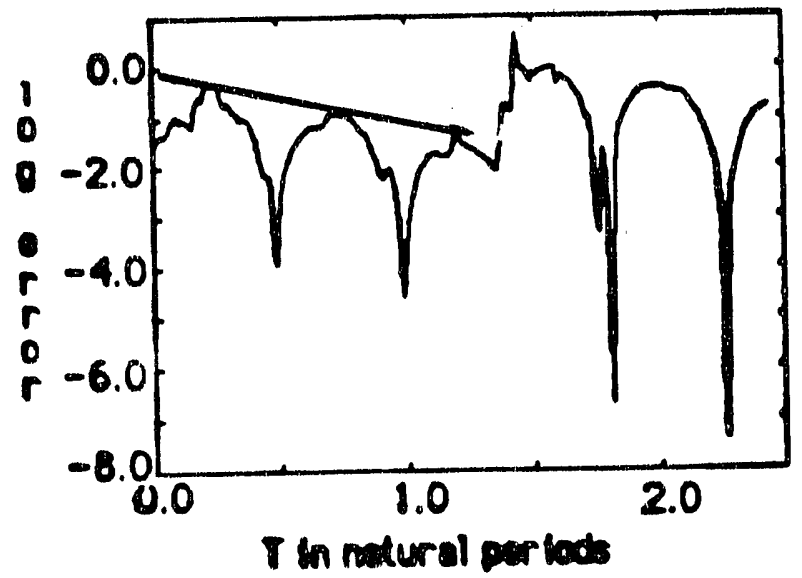

Fran sa. Error propagation in repones of bolated sccolbrometer bor gmo.z.

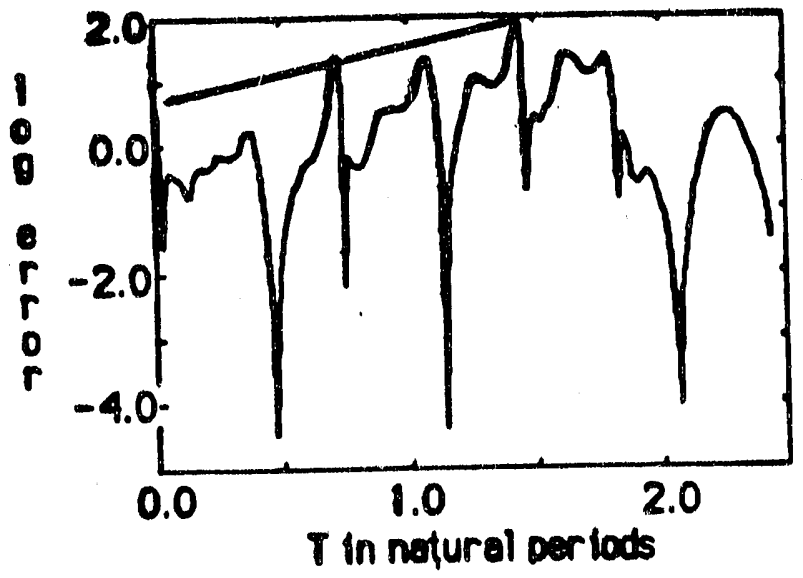

Figure ob. Error propagation in response of rolated acceleromoter for $0=3.2$.

\section{Conclusion}

The present etudy stablished a first order model for the isolated accelerometer, then normalized the model in several respects. The normalized model was used to establish paramoter regimes where chaos occurs in the system. it was shown that when the input irequency ratio, $a$ is greater than 1.75 (approximately) and the noimalized input amplitude, B. is greater than a value of 0.45 to 0.55 then chess can occur. This result indicates that a sinusoldal input whose frequency is greater than about twice the emall displaceinent natural irequency of the leolated accolerometer may cause chaotic vibrations when the actual input amplitude is oreater than about 0.5 IImes op ${ }^{2} y_{m}$. (See Eq. Ba.) This is a very severe input for typlcal leolated sccelerometer design parameters, and $n$ is anticlpated that sustained periodic inputs of this magnitude are not likely to o ..... very irequently.

Very severe Input "splkes" can, however, cccur during shock excltation, and the eecond part of this study showed what can happen when an bolated accolorometer is eubjected to shock inputs with two components. The first componont was relatively bow irequency (one that 
should be metisurable by the isolated accolerometer), and the second component is relatively high frequency (one that might cause the isolated acceleromiter to enter a chaotic regime). When the high frequency component is relatively severe, then the measurement of the low irequency component is distorted. In fact, it is shown that criterion for chaotic motion is satisfled when a severe motion is input to the system, l.e., that small "errors" In the rosponse tend to increase with time.

Additional portions of this siudy (not summarized here) show that even when the system re. sponse is not chaotic, the inferred acceleration may be substantially different from the actual low frequency component of acceleration.

The most important conclusion is that there may be some potential for chaotic motion in the isolated accelerometer when it is subjected to extreme environments. The topic merits further investigation.

\section{References}

1. Poincare, H., (1921) The Foundation of Science: Science and Method. English Translatlon, The Sciences Press, New York.

2. Parker, T., Chua, L., (1989) Practical Numerical Aloorithms for Chaotic Systems. Springer-Verlag, New York.

3. Moon, F., (1987) Chaotic Vibrations, An Introduction for Applied Scientists and Engineers John Wiley \& Sons, Now York.

4. Eubank, S., Farmer, D., (1990) "An Introduction to Chaos and Randomness," 1289 Lectures in Complex Sy atems. SFI Studies in the Sclences of Complexity, Lect. Vol. II, Ed. Erica Jen, Addison Westey.

5. Hunter, N., (1991)"Diagnosis of Nonlinear Systems Using Time Series Analysis," Proceedings of the 3rd International Machinen Monitrino and Diagnestic Conference SEM, LaS Verees.

6. Hunter, N., Nonlinear Signal Processing: Applications of Time Series Analysis to Driven Nonlinear Systems," to be published.
7. Braver, F., Nohel, J., Qualitative Theory of Ordinary Differential Equations, W. A. Benjamin, Inc., Now York.

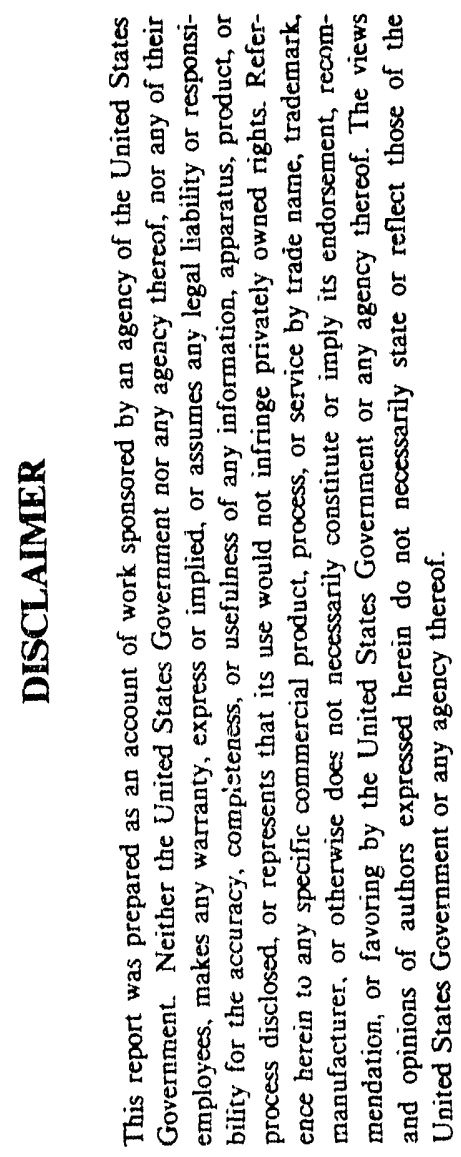



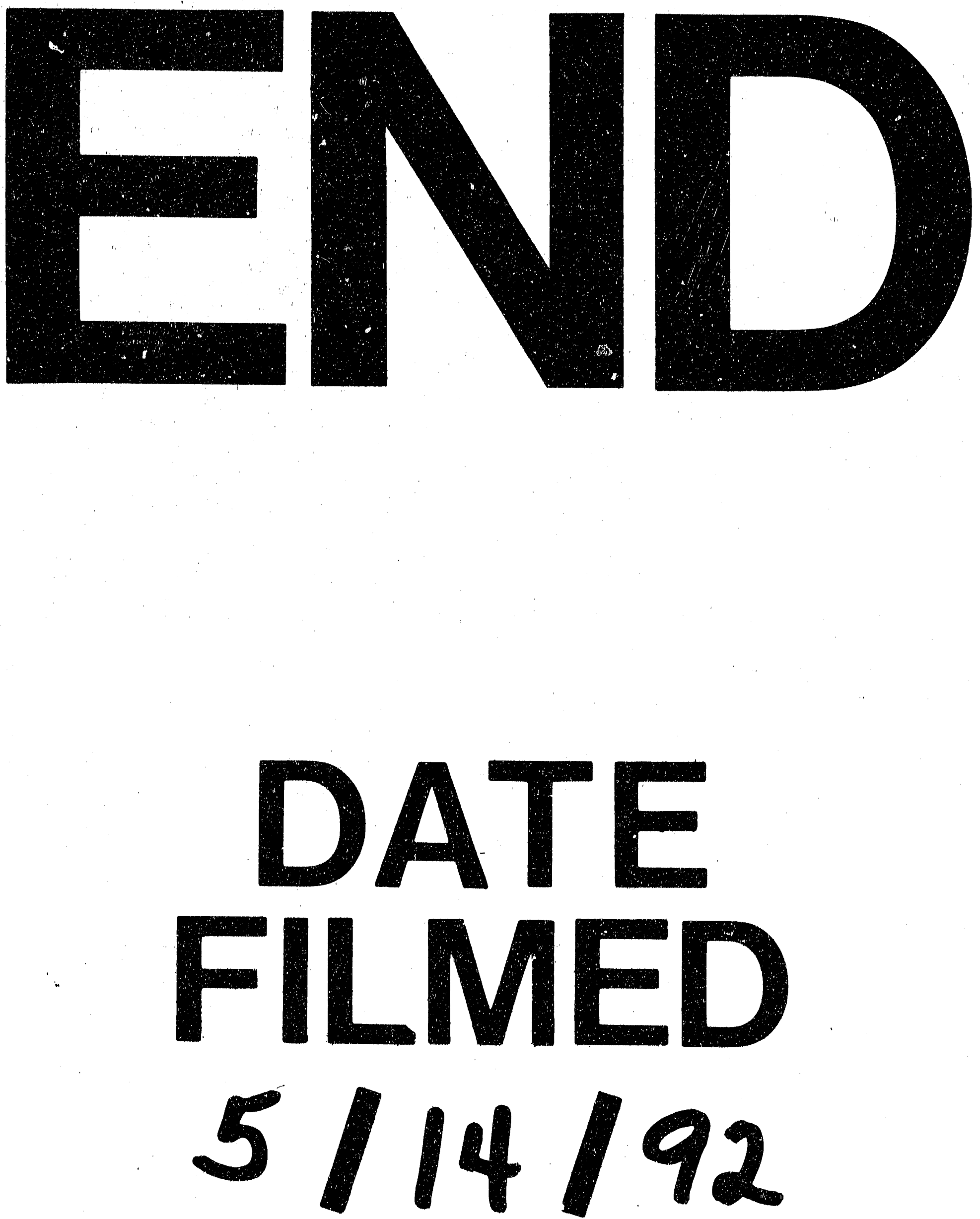
